\title{
IMPACTO DAADIÇÃO DE CAULIM NAS PROPRIEDADES FÍSICO-MECÂNICAS DO PAPEL FORMADO POR SEQUÊNCIAS ECF
}

Ivan Rodrigues dos Santos ${ }^{1 *}$, Gustavo Ventorim², José Cláudio Caraschi², Jaqueline Silveira Comelato Favaro

*Autor para correspondência: ivan@itapeva.unesp.br

RESUMO: A indústria de papel é a principal consumidora de caulim. Sua maior utilização no papel é como enchimento, pelo fato de ser mais barato do que a polpa celulósica. O processo de remoção de cor da celulose conhecido como branqueamento é determinante nas características da polpa final. Muito esforço tem sido feito para que processos de branqueamento menos tóxicos sejam viabilizados. No presente trabalho, objetivou-se avaliar a influência de três sequências de branqueamento de polpa kraft de eucalipto na retenção de caulim e nas propriedades do papel formado. A polpa industrial de eucalipto pré-deslignificada com oxigênio foi branqueada pelas sequências $\mathrm{D}(\mathrm{E}+\mathrm{P}) \mathrm{DP}$ (referência), $\mathrm{A}_{\mathrm{HT}} \mathrm{D}(\mathrm{E}+\mathrm{P}) \mathrm{DP}(\mathrm{A}-\mathrm{ECF})$ e $\mathrm{A}_{\mathrm{HT}} \mathrm{D}(\mathrm{E}+\mathrm{P}) \mathrm{P}(\mathrm{ECF}-$ Light). $\mathrm{O}$ caulim foi adicionado às polpas celulósicas após seu refino em moinho PFI para $40^{\circ} \mathrm{SR}$, correspondendo a uma quantidade mássica de $20 \%$ de caulim para cada folha formada. Os resultados apontaram que a adição de $20 \%$ de caulim apresentou retenção significativamente menor para a amostra ECF-Light, permanecendo a sequência referência igual à A-ECF; quanto maior o teor de finos, maior foi a retenção da carga; a sequência ECFLight apresentou maior custo, menor resistência ao rasgo e menor resistência interna das fibras; a lisura e a compressão não foram influenciadas pela escolha da sequência de branqueamento, tanto quanto pela adição de caulim.

Palavras-chave: polpa celulósica, caulim, aditivos.

\section{IMPACT OF KAOLIN FILLER ON PHYSICAL AND MECHANICAL PAPER PROPERTIES FORMED BY ECF PULP}

\begin{abstract}
The paper industry is the main global consumer of kaolin. It is mostly used as paper filler, due to its lower cost as compared to pulp cost. Color removal process from pulp - chemical bleaching - determines final pulp's characteristics. Effort has been made to make possible to obtain less toxic bleaching processes. This study aims to evaluate the influence of three bleaching sequences, on kaolin retention and paper properties. Industrial eucalyptus kraft pulp, oxygen pre-delignified, was bleached by the sequences $D(E+P) D P$ (reference), $A_{H T}(E+P) D P(E C F-A)$ and $A_{H T} D(E+P) P(E C F$-Light). Kaolin was added to the pulps after its refining in PFI mill to $40^{\circ} S R$, corresponding to an amount of $20 \%$ weighted kaolin for each handsheet. The results showed the addition of $20 \%$ kaolin significantly presented lower retention for ECF-Light sample, remaining reference sequence equal to A-ECF sequence; the higher the fines content, the greater the retention load; the ECF-Light sequence showed higher cost, lower tear resistance and low internal resistance of fibers; smoothness and compression were not influenced by bleaching sequence or by adding kaolin.
\end{abstract}

Keywords: cellulosic pulp, kaolin, additives.

\section{INTRODUÇÃO}

O processo de fabricação do papel à partir de polpas de eucalipto incorpora diversos complementos à massa para a fabricação de diferentes tipos de papéis. A utilização de cargas junto às fibras celulósicas durante a formação do papel é uma técnica antiga, e tornou-se imprescindível para a obtenção de papéis de uso cada vez mais específicos. O uso de materiais de enchimento para o papel pode ocasionar redução de custos e de energia, melhorar as propriedades do papel, como também de gerar papéis que apresentem uma função específica. Diversos tipos de aditivos são utilizados, e o caulim é um destes, empregado principalmente como carga de enchimento (fillers).

1 Universidade Estadual Paulista - Guaratinguetá, São Paulo, Brasil

2 Universidade Estadual Paulista - Itapeva, São Paulo, Brasil
Caulim é um mineral em forma de partícula dominante na indústria de papel, utilizado tanto para enchimento como também para revestimento de papel (BUNDY; ISHLEY, 1991). É definido como um minério composto de silicatos hidratados de alumínio, como a caulinita e a haloisita, que apresentam características especiais que permitem sua utilização no processo produtivo de papel, cerâmica, tintas, etc. Também podem ocorrer os minerais do grupo da caulinita, como diquita, nacrita, folerita, anauxita, colirita e tuesita (PRASAD et al., 1991). O caulim é primordial para reduzir a quantidade de polpa de celulose necessária para a produção do papel, além de proporcionar melhorias nas características de impressão, bem como impermeabilidade, opacidade e maciez(BUNDY; ISHLEY, 1991; LOUGHBROUGH, 1993).

Cerne, Lavras, v. 20, n. 2, p. 231-238, abr./jun. 2014 
As cargas são mais baratas que a polpa celulósica e proporcionam melhorias em algumas propriedades do papel. A escolha do tipo de carga a ser utilizada dependerá das suas características e estas determinam a viabilidade da produção do tipo de papel desejado. A retenção dessa carga na massa pode ser um fator-chave na produção de papel. O objetivo do controle de retenção é regular a passagem de sólidos (fibras, finos e cargas) junto à água nas telas de formação do papel. A retenção também contribui com a diminuição da variabilidade tanto da gramatura como do teor de cinzas ao longo do papel (ALMEIDA, 1988). A resistência do papel é inevitavelmente reduzida pela substituição de fibras por fillers, não somente porque há menos fibras no papel, o que reduz o número de ligações fibra-fibra, mas também por reduzir a área de contato entre as fibras remanescentes (LIN et al., 2007; SHEN et al., 2009). Segundo Lin et al. (2007), a quantidade de finos presentes na polpa celulósica aumenta a retenção de cargas minerais. Os autores ainda afirmam que o teor de finos contribui no aumento das propriedades de resistência, pois até certo teor, aumenta o número de ligações interfibras, além de melhorar as propriedades óticas do papel. Kinoshita et al. (2000) verificaram que as partículas pequenas tem a capacidade de ocupar os espaços vazios não preenchido pelos finos.

A sequência de branqueamento utilizada pode afetar a qualidade do papel produzido, para tanto é necessário saber se a sequência de branqueamento é um fator determinate na retenção do caulim pela massa celulósica. Objetivou-se, neste trabalho, avaliar o efeito da sequência de branqueamento na retenção de caulim, e nas propriedades físicas e mecânicas do papel formado.

\section{MATERIAL E MÉTODOS}

\subsection{Material}

Foi utilizada uma polpa kraft industrial de eucalipto proveniente de indústria celulósica, pré-deslignificada com oxigênio. As características iniciais da polpa utilizada foram: alvura de $60,7 \%$ ISO, viscosidade de $27,3 \mathrm{cP}$ e número kappa 11.

O caulim, fornecido por uma empresa do setor, é o mais utilizado industrialmente como carga pelas indústrias de celulose e papel. Apresentou as seguintes características: alvura de $88 \%$ ISO, $45 \%$ de partículas menores de $2 \mu \mathrm{m}$ e abrasividade muito baixa.

Cerne, Lavras, v. 20, n. 2, p. 231-238, abr./jun. 2014

\subsection{Branqueamento}

Foram adotadas três sequências de branqueamento, sendo elas: $\mathrm{D}(\mathrm{E}+\mathrm{P}) \mathrm{DP}$ (referência), $\mathrm{A}_{\mathrm{HT}} \mathrm{D}(\mathrm{E}+\mathrm{P}) \mathrm{DP}$ (A-ECF) e $A_{\mathrm{HT}} \mathrm{D}(\mathrm{E}+\mathrm{P}) \mathrm{P}$ (ECF-Light), onde $\mathrm{D}, \mathrm{A}_{\mathrm{HT}},(\mathrm{E}+\mathrm{P})$ e P são siglas referentes aos estágios de dióxido de cloro, hidrólise ácida em alta temperatura, extração alcalina com peróxido de hidrogênio e peróxido de hidrogênio, respectivamente. As condições gerais de branqueamento são apresentadas na Tabela 1.

Tabela 1 - Condições gerais de branqueamento.

Table 1 - General bleaching conditions.

\begin{tabular}{ccccccc}
\hline \multirow{2}{*}{ Parâmetro } & \multicolumn{6}{c}{ Estágio de branqueamento } \\
\cline { 2 - 7 } & AHT & $\mathrm{D}$ & $(\mathrm{E}+\mathrm{P})$ & $\mathrm{D}$ & $\mathrm{P}$ & $\mathrm{P}^{*}$ \\
\hline Consistência, \% & 10 & 10 & 10 & 10 & 10 & 10 \\
Temperatura, ${ }^{\circ} \mathrm{C}$ & 95 & 60 & 70 & 70 & 95 & 95 \\
Tempo, min & 120 & 30 & 60 & 180 & 120 & 120 \\
$\mathrm{H}_{2} \mathrm{O}_{2}, \mathrm{~kg} / \mathrm{t}$ & - & - & 3 & - & 6 & 20 \\
$\mathrm{NaOH}_{1} \mathrm{~kg} / \mathrm{t}$ & - & - & 10 & - & 6 & 6 \\
$\mathrm{H}_{2} \mathrm{SO}_{4}, \mathrm{~kg} / \mathrm{t}$ & 5 & 1 & - & - & - & - \\
$\mathrm{ClO}_{2}, \mathrm{~kg} / \mathrm{t}$ & - & $* *$ & - & 12 & - & - \\
Fator kappa $_{2}$ & - & 0,2 & - & - & - & - \\
$\mathrm{pH}$ final & 3,0 & 3,0 & 11,0 & 3,8 & 10,5 & 10,5 \\
\hline
\end{tabular}

*Estágio de peróxido de hidrogênio somente para a sequência ECF-Light

${ }^{*}$ Dosagem de acordo com o fator kappa. Para a sequência ECFLight foi utilizado $10 \mathrm{~kg} / \mathrm{t}$.

O estágios de branqueamento foram efetuados em sacos de polietileno com amostras representativas de polpa. $\mathrm{O}$ licor de branqueamento foi adicionado à polpa em temperatura ambiente. Terminada a reação, foram extraídas amostras de licor residual das polpas para análises de $\mathrm{pH}$. A polpa foi lavada com o equivalente a $9 \mathrm{~kg}$ de água por tonelada de polpa absolutamente seca.

As análises de número kappa, alvura e viscosidade da polpa foram efetuadas de acordo com os procedimentos descritos pelas normas TAPPI -T236 om 99, TAPPI T525 om 92 e TAPPI -T 230 om 99, respectivamente (TECHNICAL ASSOCIATION OF THE PULP AND PAPER INDUSTRY - TAPPI, 2001). Esses ensaios foram realizados em duplicata. Os custos dos reagentes de branqueamento foram calculados em US\$/t a.s. de polpa e 
os preços dos reagentes a seguir (US\$/t do produto) foram usados para calcular os custos dos reagentes químicos (base $100 \%$ de pureza) de branqueamento (US $\$ / \mathrm{kg}$ produto): dióxido de cloro $\left(\mathrm{ClO}_{2}\right)=1,0$; peróxido de hidrogênio $\left(\mathrm{H}_{2} \mathrm{O}_{2}\right)=0,85$; hidróxido de sódio $(\mathrm{NaOH})=0,50$ e ácido sulfúrico $\left(\mathrm{H}_{2} \mathrm{SO}_{4}\right)=0,08$.

\subsection{Determinação do teor de celulose da polpa branqueada}

O teor de celulose foi determinado a partir da polpa branqueada a $90 \%$ ISO, considerando-a composta de $100 \%$ holocelulose para fins práticos. Em um béquer de $100 \mathrm{~mL}$ colocou-se $1,00 \mathrm{~g}$ a.s. de holocelulose e 15,0 $\mathrm{mL}$ de hidróxido de potássio a 24,0\%. Após 15 horas sob agitação, filtrou-se a mistura em funil de vidro sinterizado $\mathrm{n}^{\circ} 1$, previamente tarado, lavando o precipitado com água destilada até a neutralidade. Em seguida, lavou-se com ácido acético 1,0\% e, por último, com etanol em excesso. A celulose obtida foi seca ao ar ambiente por 48 horas e em estufa a $105 \pm 3{ }^{\circ} \mathrm{C}$ por 30 minutos. Resfriou-se a celulose em dissecador até massa constante. Determinou-se o teor de celulose (\%Celulose) a partir da Equação 1, sendo que $\mathrm{m}^{1}$ é a massa de celulose seca, em gramas; e $\mathrm{m}_{2}$ é a massa de holocelulose seca, em gramas.

$$
\% \text { Celulose }=100\left(\mathrm{~m}_{1} / \mathrm{m}_{2}\right)
$$

\subsection{Preparação e aplicação do caulim}

Após o refino, foi adicionada à solução de polpa celulósica durante o preparo do corpo de prova (folhinha) para testes físicos, a fração correspondente em massa para que a quantidade do caulim fosse equivalente a $20 \%$ em massa do papel. O caulim foi moído em um gral com o auxílio de um pistilo. Em um béquer de 1L, adicionouse $700 \mathrm{~mL}$ de água destilada e o caulim. A mistura foi agitada por $20 \mathrm{~min}$. $\mathrm{O}$ caulim foi adicionado à polpa no desintegrador laboratorial de fibras e desintegrado a 16.000 rotações junto à solução de polpa. A retenção de caulim foi determinada pelo teor de cinzas de acordo com a norma TAPPI - T 211 om - 93. Os corpos de prova foram confeccionados de acordo com a norma TAPPI - T 205 $\mathrm{sp}-95$, com gramatura aproximada de $60 \mathrm{~g} / \mathrm{m}^{2}$.

As fibras da polpa celulósica livre de caulim foram analisadas em FQA (Fiber Quality Analyzer). Foram determinadas as seguintes dimensões: comprimento médio ponderado por comprimento de fibras, coarseness e teor de finos - fração de polpa que atravessa uma malha de 200 mesh. Isso equivale a um tamanho médio de partícula de $75 \mu \mathrm{m}$ (TAPPI $233 \mathrm{~cm} 06$ e TAPPI 261-cm-00).

\subsection{Ensaios físicos e mecânicos}

Os ensaios físicos e mecânicos foram realizados conforme os procedimentos NBR e TAPPI, indicados na Tabela 2.

Tabela 2 - Procedimentos utilizados para confecção e análise das amostras.

Table 2 - Procedures used for handsheet confection and analysis

\begin{tabular}{lc}
\hline \multicolumn{1}{c}{ Ensaio } & Procedimento \\
\hline Pasta celulósica - Refinação em PFI & NBR 14345:2004 \\
Confecção de folha para teste físico & TAPPI T205 sp - 95 \\
Teor de cinzas & TAPPI T211 om - 02 \\
Lisura (Bekk) & TAPPI T479 cm - 99 \\
Resistência à compressão (Short-span) & TAPPI T826 pm - 92 \\
Resistência ao rasgo (Elmendorf) & TAPPI T414 om - 98 \\
Resistência interna (Scott Bond) & TAPPI T569 pm - 00 \\
\hline
\end{tabular}

Esses ensaios, foram conduzidos com 10 repetições cada. Para a análise estatística dos resultados, utilizou-se análise de variância (ANOVA) e do teste de comparação de médias pelo teste de Tukey a 5\% de significância (95\% de probabilidade).

\section{RESULTADOS E DISCUSSÃO}

\subsection{Branqueamento}

Os resultados de qualidade da polpa branqueada para as três sequências de branqueamento adotadas são apresentados na Tabela 3. Determinou-se que os testes físicos e mecânicos fossem realizados para polpas com resistência à drenagem equivalente a $40^{\circ} \mathrm{SR}$. Também são apresentadas as características finais de cada polpa e o número de revoluções do refino, para a obtenção de $40^{\circ} \mathrm{SR}$.

A seletividade é a relação entre a variação do número kappa e a variação da viscosidade ( $\Delta$ kappa / $\Delta$ viscosidade) no estágio. Quanto mais seletivo é um estágio ou sequência de branqueamento, maior é sua eficiência de deslignificação e branqueamento, medida pela redução do número kappa e menor é sua agressividade às cadeias

Cerne, Lavras, v. 20, n. 2, p. 231-238, abr./jun. 2014 
poliméricas, medida pela viscosidade. Foi observado na Tabela 3, que a sequência referência apresentou maior viscosidade que as demais. Isto se deve ao fato do dióxido de cloro ser um oxidante mais seletivo que o peróxido de hidrogênio, reagindo primeiro com as ligninas presentes na polpa (RAGNAR, 2001), fazendo com que as cadeias de polissacarídeos sejam mais preservadas. A sequência referência apresentou menor custo de reagentes, quando comparada à sequência ECF-Light, conforme observado na Tabela 3, bem como a menor demanda de cloro ativo total. Esses resultados estão de acordo com Ventorim et al. (2009), pois concluíram que o estágio ácido promove uma economia de $23 \%$ no consumo do dióxido de cloro na seqüência $\mathrm{OA}_{\mathrm{HT}} \mathrm{D}(\mathrm{E}+\mathrm{P}) \mathrm{DD}$, mostrando ser um importante meio de redução de custo ou aumento da produção da polpa branqueada.

Tabela 3 - Resultados do branqueamento e características finais das amostras.

Table 3 - Bleaching results and final samples characteristics.

\begin{tabular}{|c|c|c|c|}
\hline \multirow[b]{2}{*}{ Parâmetro } & \multicolumn{3}{|c|}{ Sequências de branqueamento } \\
\hline & $\begin{array}{l}\mathrm{D}(\mathrm{E}+\mathrm{P}) \mathrm{DP} \\
\text { (Referência) }\end{array}$ & $\begin{array}{c}\mathrm{A}_{\mathrm{HT}} \mathrm{D}(\mathrm{E}+\mathrm{P}) \mathrm{DP} \\
(\mathrm{A}-\mathrm{ECF})\end{array}$ & $\begin{array}{l}\mathrm{A}_{\mathrm{HT}} \mathrm{D}(\mathrm{E}+\mathrm{P}) \mathrm{P} \\
\text { (ECF-Light) }\end{array}$ \\
\hline Alvura A. D., \%ISO & 90,5 & 90,1 & 86,6 \\
\hline $\begin{array}{l}\text { Número Kappa no } \\
\text { estágio }(\mathrm{E}+\mathrm{P})\end{array}$ & 4,4 & 3,2 & 4,8 \\
\hline Viscosidade, $\mathrm{cP}$ & 17,8 & 15,7 & 13,9 \\
\hline $\mathrm{ClO}_{2}$ como Cl${ }_{2}, \mathrm{~kg} / \mathrm{t}$ & 24,0 & 27,8 & 10,0 \\
\hline $\mathrm{H}_{2} \mathrm{O}_{2}, \mathrm{~kg} / \mathrm{t}$ & 9,0 & 9,0 & 25,0 \\
\hline $\mathrm{NaOH}, \mathrm{kg} / \mathrm{t}$ & 16,0 & 16,0 & 16,0 \\
\hline $\mathrm{H}_{2} \mathrm{SO}_{4}, \mathrm{~kg} / \mathrm{t}$ & 1,0 & 6,0 & 6,0 \\
\hline $\begin{array}{l}\text { Seletividade após } \\
\text { estágio }(\mathrm{E}+\mathrm{P})\end{array}$ & 1,29 & 1,09 & 0,92 \\
\hline Cloro ativo total, $\%$ & 4,28 & 4,66 & 6,23 \\
\hline $\begin{array}{l}\text { Custo Total de Re- } \\
\text { agentes, US\$/t }\end{array}$ & 24,9 & 26,7 & 33,5 \\
\hline Hemiceluloses, \% & 17,2 & 15,2 & 14,7 \\
\hline Teor de cinzas, $\%$ & 0,24 & 0,16 & 0,20 \\
\hline Refino, revoluções PFI & $2750^{\mathrm{a}}$ & $2750^{\mathrm{a}}$ & $2790^{\mathrm{b}}$ \\
\hline
\end{tabular}

O menor teor de cinzas nas polpas que passaram pelo estágio ácido é explicado pelo fato de que este estágio remove metais. A polpa ECF-Light apresentou maior teor de cinzas que a polpa A-ECF, porque teve uma maior dosagem de peróxido de hidrogênio no último estágio e, consequentemente, uma maior dosagem de soda. Nem todo sódio é removido da polpa durante a lavagem, refletindo, assim, no resultado de teor de cinzas para essa polpa.
Segundo ainda a Tabela 3, a polpa referência apresentou maior teor de hemiceluloses. As polpas que tiveram estágio ácido no branqueamento apresentaram, entre elas, uma diferença de $0,5 \%$ em seus teores. A polpa que apresentou um menor teor de hemiceluloses foi a ECF-Light, pela maior degradação dos polissacarídeos no estágio de hidrólise ácida e a maior carga de peróxido de hidrogênio na polpa ECF-Light.

Verificou-se que, tanto a sequência referência quanto a sequência A-ECF necessitaram de 2750 revoluções PFI cada, para atingir $40^{\circ} \mathrm{SR}$, enquanto que a sequência ECF-Light necessitou de 2790 revoluções para atingir o mesmo nível de refinação. $\mathrm{O}$ estudo de Tanaka et al. (2001), verificou que tanto o refino quanto a adição de caulim na polpa apresentam ambos o mesmo efeito de redução da maioria das propriedades mecânicas da polpa kraft. Embora essa relação não seja linear, ambos influenciam o grau de ligação da rede da fibra e assim alteram a configuração elástica da ligação.

Pode-se atribuir a esse maior número de revoluções o menor percentual de hemiceluloses que esta sequência apresenta, equivalente a $14,7 \%$ de teor de hemiceluloses, enquanto a sequência referência e ECF-Light apresentou 17,2 e 15,2 respectivamente, conforme exposto na Tabela 3 , pois as hemiceluloses facilitam o refino. Segundo Moldes et al. (2010), a degradação das hemiceluloses consequente da remoção de ácidos hexenurônicos, durante o estágio ácido, ocasiona a redução da drenabilidade das polpas. O mesmo ocorreu para a polpa ECF-Light, quando comparada com a sequência referência.

Na Tabela 4, verificam-se os parâmetros obtidos para análise de fibras. Foi observado que tanto a sequência A-ECF como a sequência ECF-Light apresentaram menores dimensões de fibra do que a sequência referência. Pode-se relacionar esse resultado à degradação causada pelo estágio ácido adotado nessas sequências. O parâmetro de comprimento de fibras apresentou diferenças de 5,5\% para a sequência $\mathrm{A}-\mathrm{ECF}$ e de $7,4 \%$ para a sequência ECF-Light quando comparado com o comprimento da fibra obtida pela sequência referência. A diferença de comprimento de fibras para essas duas sequências é explicada pela maior degradação ocasionada pela hidrólise ácida e pela alta carga de peróxido de hidrogênio utilizada na sequência ECF-Light, causando extensivo ataque aos polissacarídeos. A largura apresentou diferença maior entre a sequência referência e a ECF-Light, sendo 9,3\% inferior.

Um reflexo da baixa seletividade do peróxido de hidrogênio é o maior teor de finos gerado na sequência ECF-Light - 12,2\% - quando comparado ao teor de finos gerado pela sequência referência, pois quanto maior a degradação da polpa, maior é o teor de finos gerado. A sequência com estágio ácido apresentou 9,8\% de finos

Cerne, Lavras, v. 20, n. 2, p. 231-238, abr./jun. 2014 
em sua polpa final perante os $7,5 \%$ de finos gerados pela sequência referência. Liimatainen et al. (2008) reportaram que o teor de finos é extremamente importante para a retenção de fillers no papel e verificaram que quanto maior o teor de finos, maior é a retenção de fillers até certo ponto. A partir desse ponto, a retenção de fillers começa a diminuir. De acordo com Lin et al. (2007), os finos melhoram muito as propriedades de resistência do papel, mas diminuem a drenagem. Já, os fillers não retardam a drenagem, apresentam um efeito positivo sobre as propriedades óticas, no entanto, diminuem a resistência do papel. Ainda observaram que a retenção de fillers é menor em comparação com a retenção de finos.

Tabela 4 - Análise de fibras.

Table 4 - Fiber analysis.

\begin{tabular}{cccc}
\hline \multirow{2}{*}{ Parâmetro } & \multicolumn{3}{c}{ Sequências } \\
\cline { 2 - 4 } & $\mathrm{D}(\mathrm{E}+\mathrm{P}) \mathrm{DP}$ & $\mathrm{A}_{\mathrm{HT}} \mathrm{D}(\mathrm{E}+\mathrm{P}) \mathrm{DP}$ & $\mathrm{A}_{\mathrm{HT}} \mathrm{D}(\mathrm{E}+\mathrm{P}) \mathrm{P}$ \\
\hline Comprimento, $\mathrm{mm}$ & 0,54 & 0,53 & 0,50 \\
Largura, $\mathrm{mm}$ & 130,20 & 123,40 & 120,90 \\
Coarseness, $\mathrm{mg} / 100 \mathrm{~m}$ & 8,90 & 8,40 & 8,10 \\
Teor de finos, $\%$ & $7,50 \mathrm{a}$ & $9,80 \mathrm{~b}$ & $12,20 \mathrm{c}$ \\
\hline
\end{tabular}

\subsection{Retenção e resistência mecânica do papel}

A retenção de caulim foi menor para a sequência referência e maior para a sequência ECF-Light, conforme verifica-se na Figura 1, indicando o percentual de caulim retido com relação à quantidade de caulim adicionada antes do desaguamento para cada folha. Lin et al. (2007) reportaram que além do teor de finos, outro fator responsável pela retenção de fillers é o tamanho das fibras, sendo que um menor tamanho de fibra tende a diminuir os espaços na superfície do papel, resultando assim em uma maior retenção. Para uma mesma espécie de madeira, a sequência de branqueamento adotada também influi, tanto no diâmetro como no comprimento das fibras. Dessa maneira, atribuiu-se maior retenção das sequências que sofreram uma maior degradação, avaliada pela menor viscosidade, ao fato destas apresentarem menor comprimento de fibra e maior teor de finos, implicando, assim, em maior número de ligações entre fibras.

É conhecido que os finos desempenham papel de material de ligação entre fibras e de preenchimento entre fibras longas, sendo que em ausência de agentes aglutinantes, as fibras mais longas têm capacidade de reter mais fillers, pois se depositam na tela formando um filtro, enquanto os finos podem reter mais fillers por meio de ligações químicas (LIIMATAINEN et al., 2008).

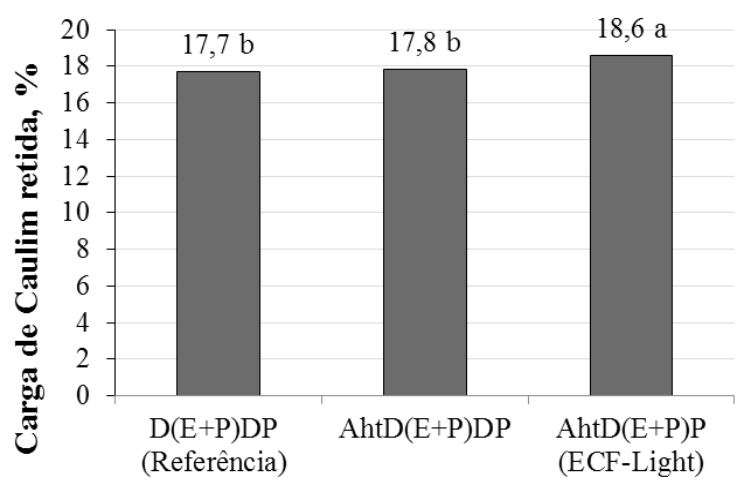

Figura 1 - Retenção de caulim para adição de $20 \%$ na massa. Médias seguidas por uma mesma letra em um mesmo conjunto de barras não apresentam diferença significativa pelo teste de Tukey a 5\% de probabilidade.

Figure 1 - Retention of 20\% in mass of kaolin. Means followed by the same letter do not significantly differ by the Tukey's test at 5\% probability level.

Na Figura 2, verificam-se os resultados dos ensaios de caracterização do papel livre de aditivos $(0 \%)$ e também com adição de $20 \%$ de caulim. Na Figura 2a, para o ensaio de lisura, foi verificado que a adição de caulim não influenciou a lisura das amostras, pois para uma mesma sequência, a média foi considerada estatisticamente tanto para $0 \%$ como para $20 \%$ de caulim. A sequência ECF-Light com $20 \%$ de caulim apresentou lisura estatisticamente igual referência com $20 \%$ e esta, por sua vez, igual a referência com $0 \%$ e ambas amostras da sequência A-ECF. Portanto, verifica-se que a escolha da sequência de branqueamento não influenciou a lisura do papel formado, tanto quanto à adição de $20 \%$ de carga de caulim. As elevadas lisuras encontradas em todas as amostras se devem à melhor consolidação do papel, em razão do teor de finos produzidos durante o refino, pois estes preenchem os poros, formando uma folha com alta lisura (KROGERUS; FAGERHOLM, 2002; LEE et al., 2011).

Pela Figura $2 b$, verifica-se que não houve diferença estatística para o ensaio de compressão pelo método Shortspan. Ou seja, a sequência de branqueamento e a presença de caulim não influenciaram o índice de compressão do papel, mesmo a retenção de caulim sendo diferente para as três sequências.

Cerne, Lavras, v. 20, n. 2, p. 231-238, abr./jun. 2014 

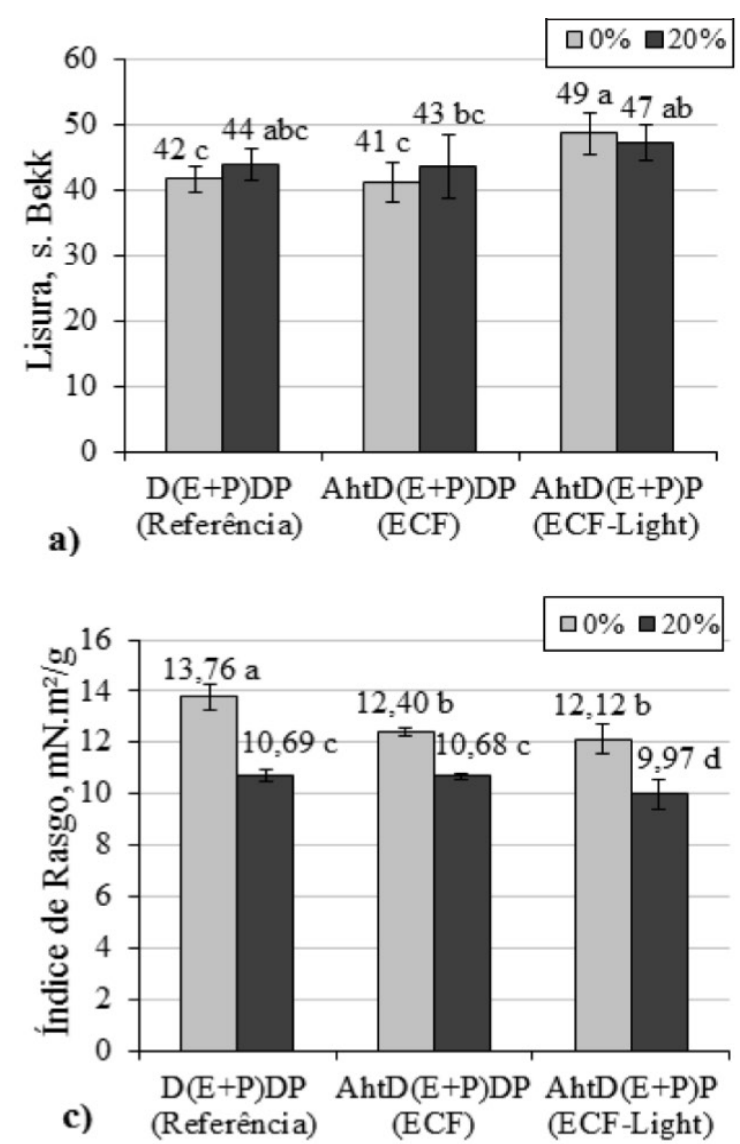
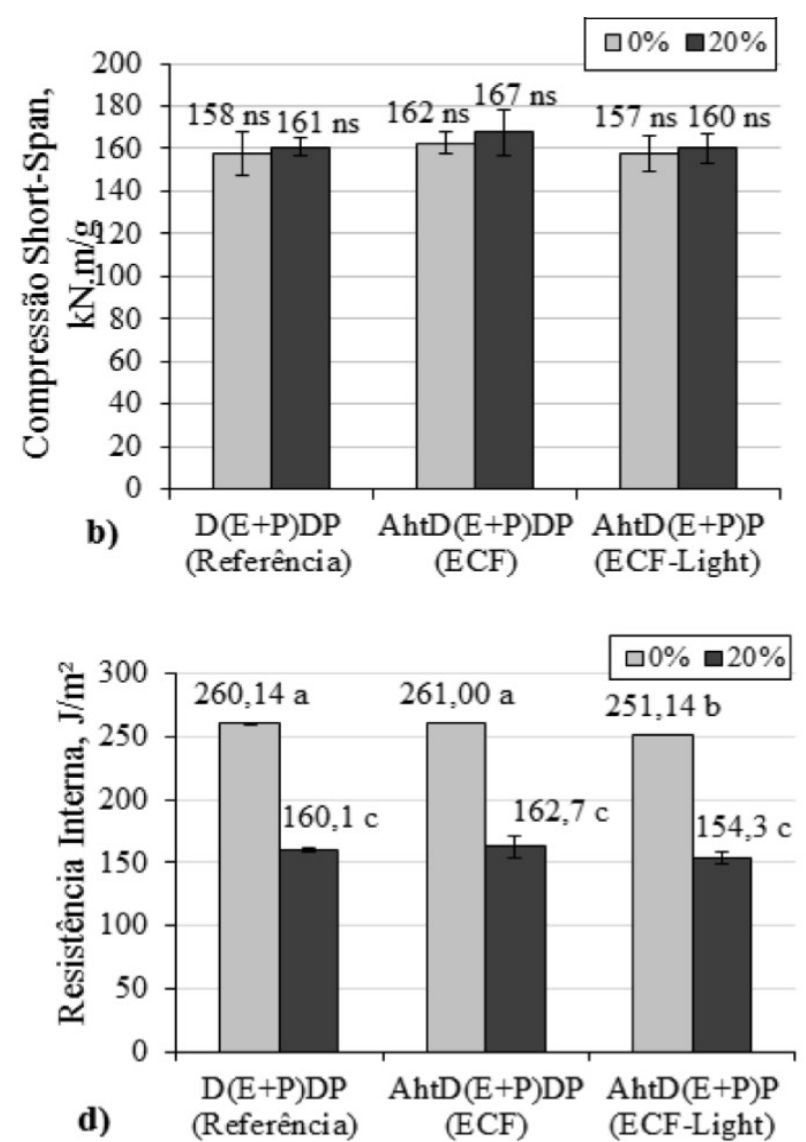

Figura 2 - Ensaios de a) lisura Bekk, b) resistência à compressão short-span, c) resistência ao rasgo e d) resistência interna das fibras, para as três sequências de branqueamento, com adição de $0 \%$ e $20 \%$ de caulim. Médias seguidas por uma mesma letra em um mesmo conjunto de barras não apresentam diferença significativa pelo teste de Tukey a $5 \%$ de probabilidade.

Figure 2 - a) Bekk smoothness, b) short-span compression resistance, c) tear resistance and d) the internal resistance of fibers, for the three bleaching sequences, on the addition of $0 \%$ and $20 \%$ of kaolin. Means followed by the same letter do not significantly differ by the Tukey's test at 5\% probability level.

A resistência ao rasgo, no entanto, foi afetada pela adição de caulim e também pela escolha da sequência de branqueamento. Foi verificado, na Figura 2c, que a sequência referência e o estágio ácido foram considerados estatisticamente iguais e maiores que a sequência ECFLight, para as amostras contendo $20 \%$ de caulim. Esse menor valor encontrado pode ser referente ao menor comprimento de fibras, pois, segundo a literatura (KINOSHITA et al., 2000; PAGE, 1994; SETH; PAGE, 1988) a resistência ao rasgo é fortemente influenciada pelo comprimento das fibras do papel. Kinoshita et al. (2000) verificaram que a resistência ao rasgo do papel diminui com o incremento de fillers na massa, pois, ao aumentar a densidade de partículas na folha, diminuem-se as ligações entre fibras, em decorrência da redução da área de contato entre elas, o que, em seguida, diminui a resistência das ligações de contato.

A resistência interna das fibras, medida pelo método "Scott", conforme Figura 2d, foi maior para as amostras livres de caulim. Os autores Bundy e Ishley (1991) verificaram que a presença de caulim como preenchimento do papel diminui, substancialmente, a resistência entre as fibras, em razão da baixa interação das fibras com o caulim na ausência de agentes aglutinantes. $\mathrm{O}$ aumento do volume específico aparente do papel causado pela presença do caulim na amostra é o principal fator para a redução

Cerne, Lavras, v. 20, n. 2, p. 231-238, abr./jun. 2014 
de resistência do papel, considerado por Gil (1988 citado por BUNDY; ISHLEY, 1991). A resistência interna da amostra $0 \%$ da sequência ECF-Light foi estatisticamente considerada inferior às amostras $0 \% \mathrm{~A}$-ECF e $0 \%$ referência. $\mathrm{O}$ menor entrelaçamento das fibras causado pela fragmentação dos polissacarídeos, medida pela baixa seletividade e baixa viscosidade dessa sequência, refletiu em menor resistência interna da sequência ECF-light.

\section{CONCLUSÕES}

Avaliando-se a escolha da sequência de branqueamento na retenção de $20 \%(\mathrm{~m} / \mathrm{m})$ de caulim, pode-se inferir que:

- a adição de $20 \%(\mathrm{~m} / \mathrm{m})$ de caulim apresentou retenção significativamente maior para a amostra ECFLight, permanecendo a sequência referência igual a A-ECF;

- quanto maior o teor de finos, maior é a retenção da carga;

- a sequência ECF-Light apresentou maior custo, menor resistência ao rasgo e menor resistência interna das fibras;

- a lisura e a compressão não foram influenciadas pela escolha da sequência de branqueamento, tanto quanto pela adição de $20 \%$ de caulim;

- a resistência ao rasgo foi influenciada pelo comprimento da fibra e pela adição de caulim nas amostras.

\section{AGRADECIMENTOS}

Ao Conselho Nacional de Desenvolvimento Científico e Tecnológico (CNPq), pelo apoio financeiro recebido.

\section{REFERÊNCIAS}

ALMEIDA, M. L. O. d'. Celulose e papel: tecnologia da fabricação da pasta celulósica. 2. ed. São Paulo: IPT/SENAI, 1988. v. 1, 319 p.

BUNDY, W. M.; ISHLEY, J. N. Kaolin in paper filling and coating. Applied Clay Science, Amsterdam, v. 5, p. 397-420, 1991.

KINOSHITA, N.; KATSUZAWA, H.; NAKANO, S.; MURAMATSU, H.; SUZUKI, J.; IKUMI, Y.; TOYOTAKE, Y. Influence of fiber length and filler particle size on pore structure and mechanical strength of filler-containing paper. The Canadian Journal of
Chemical Engineering, Ottawa, v. 78, n. 5, p. 974-982, 2000.

KROGERUS, B.; FAGERHOLM, K. Fines from different pulps compared by image analysis. Nordic Pulp and Paper Research Journal, Copenhagen, v. 17, n. 4, p. 440-444, 2002.

LEE, H.; NAMB, W. S.; SOHN, S. D.; PAIK, K. H. Effect of different types of fines on the properties of recycled chemical pulp. Journal of Industrial and Engineering Chemistry, Washington, v. 17, n. 1, p. 100-104, 2011.

LIIMATAINEN, H.; TAIPALE, T.; HAAPALA, A.; NIINIMÄKI, J. Influence of mechanical pulp fines on clay retention. TAPPI Journal, Atlanta, v. 7, n. 12, p. 10-16, 2008.

LIN, T.; YIN, X.; RETULAINEN, E.; NAZHAD, M. Effect of chemical pulp fines on filler retention and paper properties. Appita Journal, Atlanta, v. 60, n. 6, p. 469-473, 2007.

LOUGHBROUGH, R. Kaolin producers move up market. Industrial Minerals, Oxford, n. 313, p. 51-69, 1993.

MOLDES, D.; CADENA, E. M.; VIDAL, T. Biobleaching of eucalypt kraft pulp with a two laccasemediator stages sequence. Bioresource Technology, Essex, v. 212, p. 6924-6929, 2010.

PAGE, D. H. A note on the mechanism of tearing strength. TAPPI Journal, Atlanta, v. 3, n. 77, p. 201203, 1994.

PRASAD, M. S.; REID, K. J.; MURRAY, H. H. Kaolin: processing, properties and applications. Applied Clay Science, Amsterdam, v. 6, p. 87-119, 1991.

RAGNAR, M. On the importance of the structural composition of pulp for the selectivity of ozone and chlorine dioxide bleaching. Nordic Pulp and Paper Research Journal, Copenhagen, v. 16, n. 1, p. 72-79, 2001.

SETH, R. S.; PAGE, D. H. Fiber properties and tearing resistance. TAPPI Journal, Atlanta, v. 2, n. 71, p. 103107, 1988.

SHEN, J.; SONG, Z.; QIAN, X.; LIU, W. Modification of papermaking grade fillers: a brief review.

Bioresources, Oxford, v. 3, n. 4, p. 1190-1209, 2009.

Cerne, Lavras, v. 20, n. 2, p. 231-238, abr./jun. 2014 
TANAKA, A.; HILTUNEN, E.; KETTUNEN, H.;

NISKANEN, K. Inter-fiber bonding effects of beating, starch or filler. Nordic Pulp and Paper Research

Journal, Copenhagen, v. 16, n. 4, p. 306-312, 2001.

TECHNICAL ASSOCIATION OF THE PULP AND PAPER INDUSTRY. TAPPI test methods. Atlanta:

Technical Divisions and Committees, 2001.

VENTORIM, G.; CARASCHI, J. C.; COLODETTE, J. L.; GOMIDE, J. L. A influência dos ácidos HexA's no rendimento e na branqueabilidade da polpa kraft. Química Nova, São Paulo, v. 32, n. 2, p. 373-377, mar./ abr. 2009.

Recebido: 16 de outubro de 2012; aceito: 23 de agosto de 2013.

Cerne, Lavras, v. 20, n. 2, p. 231-238, abr./jun. 2014 